\title{
Erratum
}

\section{Erratum to "Existence of Solutions for Generalized Vector Quasi-Equilibrium Problems by Scalarization Method with Applications"}

\author{
De-ning Q $\mathbf{u}^{1,2}$ and Cao-zong Cheng ${ }^{1}$ \\ ${ }^{1}$ College of Applied Science, Beijing University of Technology, Beijing 100124, China \\ ${ }^{2}$ College of Mathematics, Jilin Normal University, Siping, Jilin 136000, China
}

Correspondence should be addressed to De-ning Qu; qudening@126.com

Received 14 June 2013; Accepted 20 August 2013

Copyright () 2013 D.-n. Qu and C.-z. Cheng. This is an open access article distributed under the Creative Commons Attribution License, which permits unrestricted use, distribution, and reproduction in any medium, provided the original work is properly cited.

We found a mistake in Example 15 in the published paper after its publication. The corrected version is as follows.

Example 15. Let $X=Y=Z=\mathbb{R}, E=\mathbb{R}_{+}$, and $F=$ $[-10,10] \subset Z$, and let $e(x)=1$, for all $x \in E$, and $C(x)=\mathbb{R}_{+}$, for all $x \in E$.

(1) Define

$$
G(u)= \begin{cases}{[u, u+1],} & u \in(0,10] \\ \{0\}, & u \in[-10,0]\end{cases}
$$

Evidently, $G$ is not usc on $F$. After simply calculating,

$$
\xi_{G}(x, u)= \begin{cases}u+1, & x \in E, \quad 0<u \leq 10 \\ 0, & x \in E, \quad-10 \leq u \leq 0\end{cases}
$$

$\xi_{G}$ is not usc on $E \times F$ due to the fact that $\{(x, u) \in E \times F$ : $\xi(x, u) \geq 1\}=\mathbb{R}_{+} \times(0,10]$ is not closed.

(2) Consider the following mapping:

$$
G(u)= \begin{cases}{[u, u+1],} & u \in[0,10], \\ \{0\}, & u \in[-10,0) .\end{cases}
$$

Obviously, $G$ is not lsc on $F$. Also, $\xi_{G}$ fails to be lsc on $E \times F$, where

$$
\xi_{G}(x, u)= \begin{cases}u+1, & x \in E, \quad 0 \leq u \leq 10 \\ 0, & x \in E, \quad-10 \leq u<0\end{cases}
$$

Furthermore, the following misprints should be noted.

With regard to the conceptions of $D$-closeness and $D$ bounded, in page 1, the correct text should be: $A$ is called $D$ closed [11] if $A+c l D$ is closed and $D$-bounded [11] if for each neighborhood $U$ of zero in $Y$, there exists $\lambda>0$ such that $A \subset \lambda U+D$.

In page 2 , left, line 5 , the correct text should be: "Incidentally, every TVS such that any singleton is closed is Hausdorff (see [12])."

In page 2 , right, line -18 , the correct text should be: "From now on, unless otherwise specified, let $X, Y$ and $Z$ be Hausdorff real TVSs and ..." 


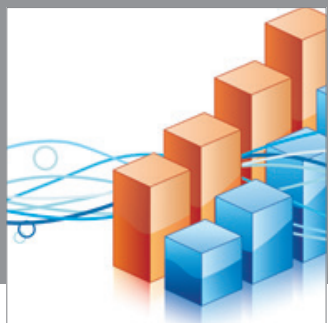

Advances in

Operations Research

mansans

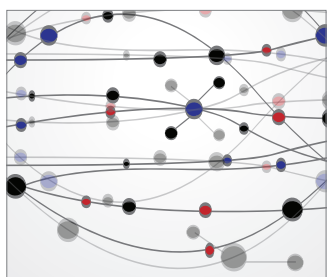

The Scientific World Journal
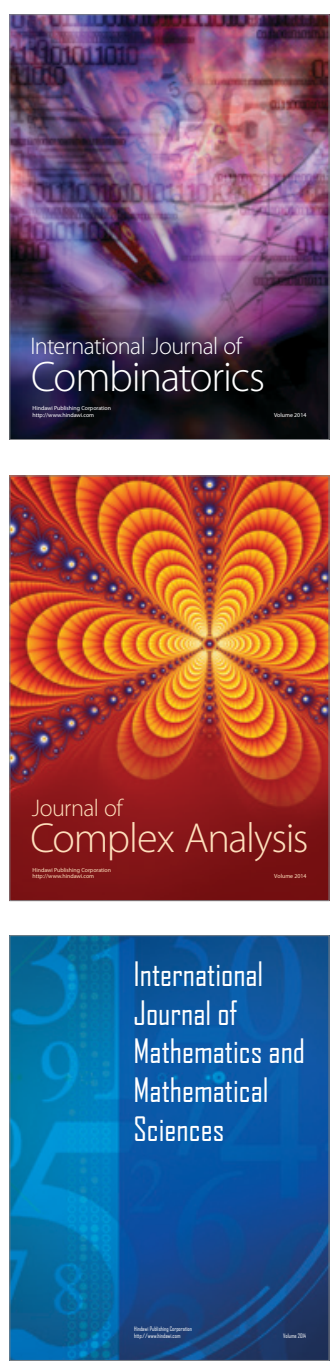
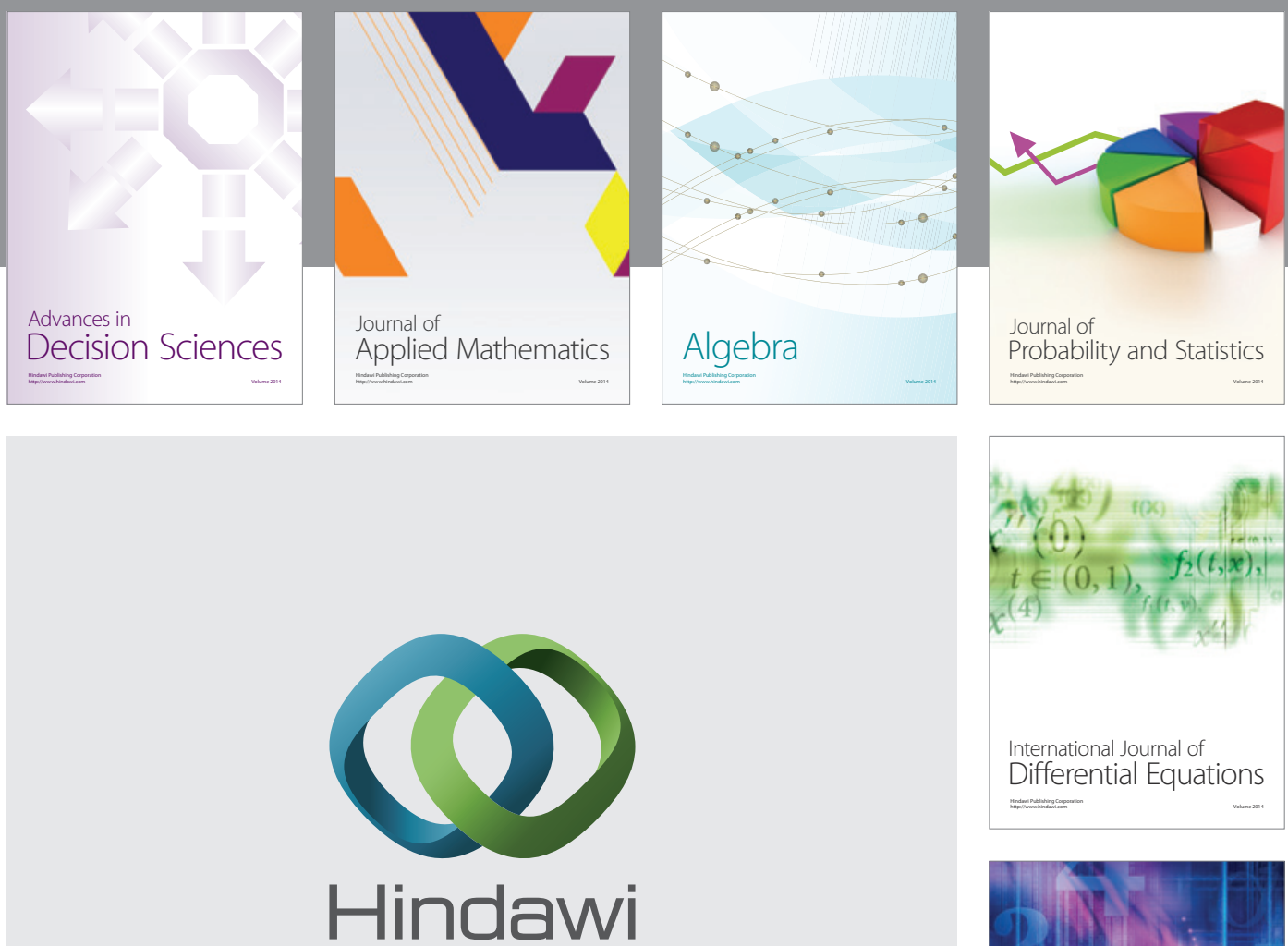

Submit your manuscripts at http://www.hindawi.com
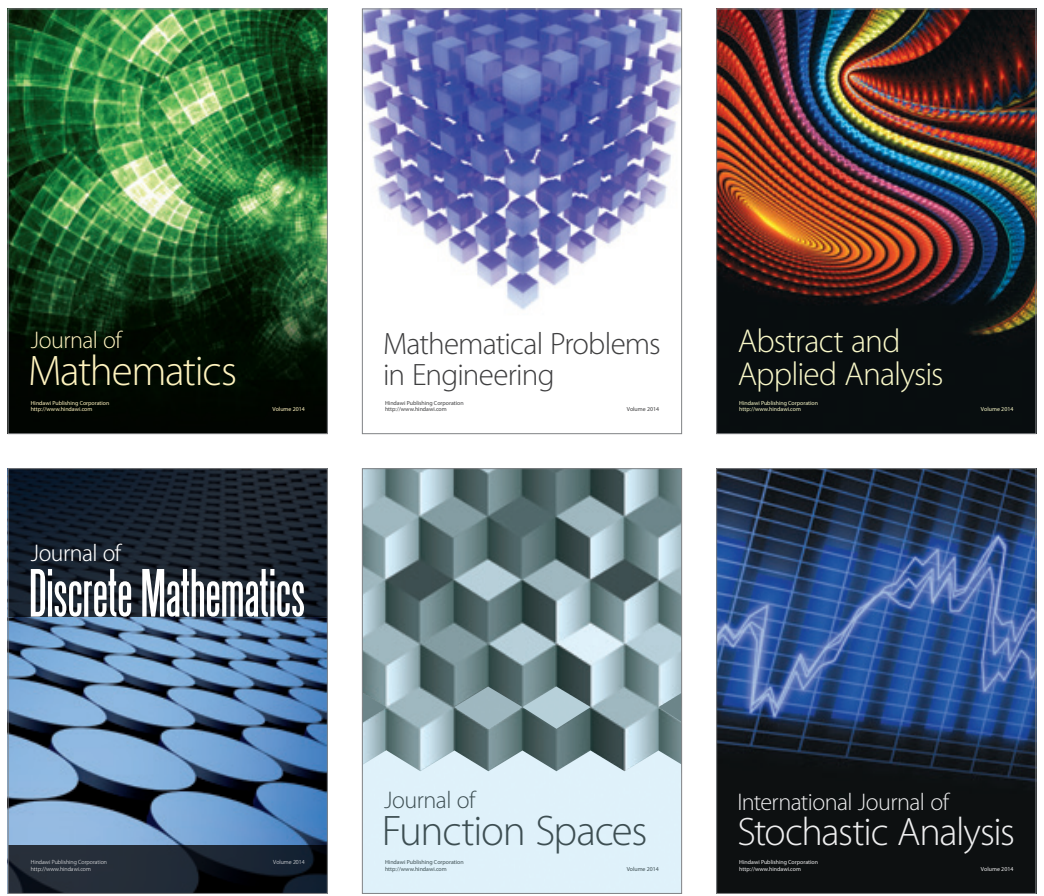

Journal of

Function Spaces

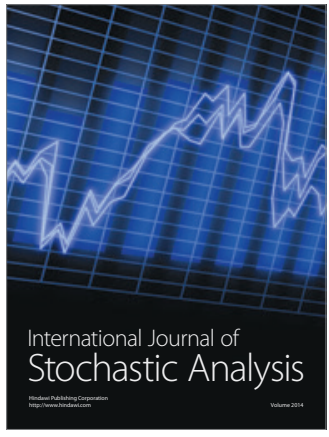

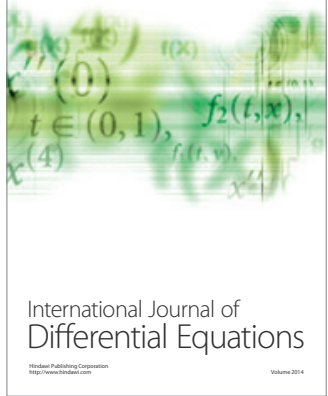
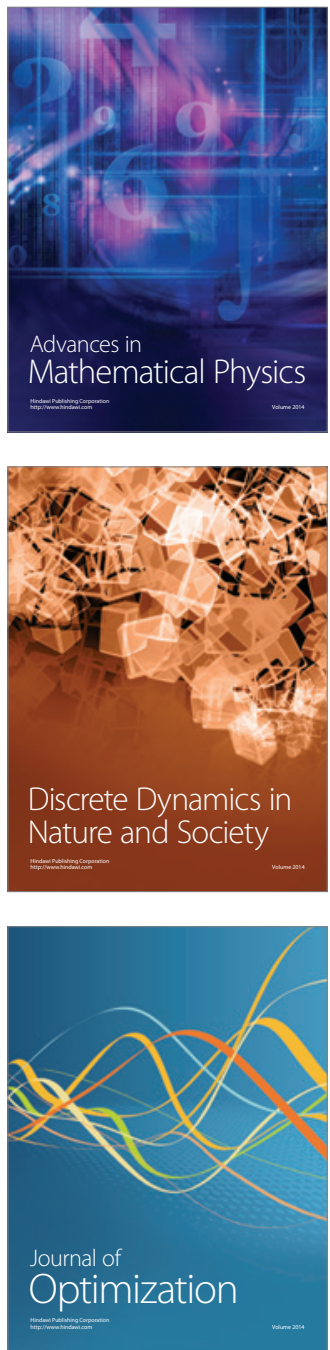